\title{
Common variants in MAEA gene contributed the susceptibility to osteoporosis in Han Chinese postmenopausal women
}

\author{
Xuan Cai ${ }^{1}$ (D) Jun Dong ${ }^{1}$, Teng Lu', Liqiang Zhi ${ }^{2}$ and Xijing $\mathrm{He}^{1^{* *}}$
}

\begin{abstract}
Background: Osteoporosis (OP) is a complex bone metabolism disorder characterized by the loss of bone minerals and an increased risk of bone fracture. A recent study reported the relationship of the macrophage erythroblast attacher gene (MAEA) with low bone mineral density in postmenopausal Japanese women. Our study aimed to investigate the association of MAEA with postmenopausal osteoporosis (PMOP) in Han Chinese individuals.

Methods: A total of 968 unrelated postmenopausal Chinese women comprising 484 patients with PMOP and 484 controls were recruited. Four tag single nucleotide polymorphisms (SNPs) that covered the gene region of MAEA were chosen for genotyping. Single SNP and haplotypic association analyses were performed, and analysis of variance was conducted to test the correlation between blood MAEA protein level and genotypes of associated SNPS.

Results: SNP rs6815464 was significantly associated with the risk of PMOP. The C allele of rs6815464 was strongly correlated with the decreased risk of PMOP in our study subjects (OR[95\% CI] $=0.75[0.63-0.89], P=0.0015)$. Significant differences in MAEA protein blood levels among genotypes of SNP rs6815464 were identified in both the PMOP ( $F=6.82, P=0.0012)$ and control groups ( $F=11.5, P=0.00001)$. The $C$ allele was positively associated with decreased MAEA protein levels in blood.

Conclusion: This case-control study on Chinese postmenopausal women suggested an association between SNP rs6815464 of MAEA and PMOP. Further analyses showed that genotypes of SNP rs6815464 were also associated with the blood level of MAEA protein.
\end{abstract}

Keywords: Postmenopausal osteoporosis, Single nucleotide polymorphisms, Genetic association, Case-control study

\footnotetext{
* Correspondence: drxhxjtu@163.com

'Department of Orthopaedic Surgery, The Second Affiliated Hospital of Xi'an Jiaotong University, No. 157 Xiwu Road, Xi'an 710004, China

Full list of author information is available at the end of the article
}

C C The Author(s). 2021 Open Access This article is licensed under a Creative Commons Attribution 4.0 International License, which permits use, sharing, adaptation, distribution and reproduction in any medium or format, as long as you give appropriate credit to the original author(s) and the source, provide a link to the Creative Commons licence, and indicate if changes were made. The images or other third party material in this article are included in the article's Creative Commons licence, unless indicated otherwise in a credit line to the material. If material is not included in the article's Creative Commons licence and your intended use is not permitted by statutory regulation or exceeds the permitted use, you will need to obtain permission directly from the copyright holder. To view a copy of this licence, visit http://creativecommons.org/licenses/by/4.0/. The Creative Commons Public Domain Dedication waiver (http://creativecommons.org/publicdomain/zero/1.0/) applies to the data made available in this article, unless otherwise stated in a credit line to the data. 


\section{Background}

Osteoporosis (OP) is a complex bone metabolism disorder characterized by loss of bone minerals and increased risk of a bone fracture [1]. It is estimated that approximately $2 \%$ to $8 \%$ of males and $9 \%$ to $38 \%$ of females are affected in industrialized countries [2]. Early epidemiological studies indicated that the incidence of OP increases with age [3]; $15 \%$ of Caucasians in their $50 \mathrm{~s}$ and $70 \%$ of those over 80 are affected [2]. In addition, the risk OP in women is approximately 3 times higher than in men $[4,5]$. OP and fractures severely affect the quality of life of a senior individual and generate great economic and psychological burdens for the patients and their families. Imbalance between bone formation and bone resorption is the basic mechanism for all osteoporosis [6]. Multiple previous studies have indicated that OP is a complex disorder caused by both genetic and environmental factors [7-10]. Based on familial samples, the heritability of osteoporosis and bone mineral density (BMD) has been estimated to be $50 \%-60 \%$ [11].

The genome-wide association study (GWAS) design has accelerated the discovery of gene-phenotype associations for complex disorders. GWAS focusing on osteoporosis and BMD have been intensively conducted in the last decade. At least 42 publications on BMD- and osteoporosis-related phenotypes have been published, and more than 200 association signals derived from approximately 90 loci that achieved genome-wide significance have been reported [12]. A large proportion of these loci are located within five major bone metabolism-related pathways, including the mesenchymal cell differentiation, WNT, NOTCH, Hedgehog and OPG-RANKRANKL signaling pathways [10]. Despite hundreds of association signals discovered by large-scale GWAS, all of these identified variants together could only explain $10-20 \%$ of the variance in bone phenotypes [10]. Therefore, more population-based studies are still needed to explore these underlying susceptible genes.

Gene macrophage erythroblast attacher (MAEA) is located on chromosome 4p16.3 and is expressed in a wide range of human cells, including osteoblasts and osteoclasts, which are important for bone metabolism. The encoded protein of MAEA is an integral membrane protein which mediates the attachment of erythroblasts to macrophages. A recent cross-sectional study suggested an association between single nucleotide polymorphism (SNP) rs6815464, a genetic polymorphism of MAEA, and low BMD of the total hip but not of the lumbar spine or femoral neck in Japanese women [13]. However, largescale and well-designed replication studies in other populations are still needed to confirm this association signal.

In the present study, we aimed to investigate the association of genetic polymorphisms of MAEA and postmenopausal osteoporosis (PMOP) based on samples of Chinese women with PMOP and controls. To determine if serum MAEA levels associated with risk SNPs for osteoporosis, blood levels of MAEA protein were also tested.

\section{Methods}

\section{Study subjects}

A total of 968 postmenopausal women comprising 484 patients with PMOP and 484 controls were recruited from the Second Affiliated Hospital of Xi'an Jiaotong University (Xi'an, China) and Honghui Hospital of Xi'an Jiaotong University (Xi'an, China) from January 2014 to March 2018. For each PMOP case, control with same gender and \pm 3 years of age was matched. Only patients with primary osteoporosis were enrolled in the present study. Patients with secondary or idiopathic OP were not included. Individuals with a history of medication for the treatment of PMOP or medication known to affect bone metabolism within 6 months were excluded from this study. In addition, only individuals with BMI $\leq 27$ were included. To control the genetic background of our study subjects, only individuals with no migration history within the previous three generations were enrolled. Diagnosis of PMOP was made based on the BMD at the lumbar spine (L2-4) and femoral neck. Dual-energy X-ray absorptiometry (Lunar Expert 1313, Lunar Corp., USA) was used to estimate the BMD. Participants who had a $\mathrm{T}$ score $<-2.5 \mathrm{SD}$ were classified as OP. This study was approved by the Medical Ethics Committee of Xi'an Jiaotong University Health Science Center. Written informed consent was provided by all study subjects.

\section{SNP selection and genotyping}

To maximize the genetic information coverage, we selected tag SNPs for genotyping. We established the following selection criteria: (i) the selection of SNPs located within up/downstream $10 \mathrm{~kb}$ of MAEA with minor allele frequency (MAF) greater than 0.05; (ii) the selection of tag SNPs using $\mathrm{r} 2 \geq 0.6$ as the criterion. Chinese Han Beijing data in the 1000 Genomes database were utilized as a reference panel. Finally, 4 intronic SNPs were chosen for genotyping (Supplemental Table S1). Genomic DNA of our study subjects was extracted from peripheral blood leukocytes using a DNA extraction kit (Tiangen Biotech Co. Ltd, Beijing, China) according to the protocol provided by the manufacturer. SNP genotyping was performed based on the Sequenom MassARRAY platform (Sequenom, San Diego, CA, USA) according to the manufacturer's protocol. Genotyping technicians were blinded to the labels of cases and controls during the experimental process. Fifty study subjects were randomly chosen for replication to evaluate the quality of genotyping 
experiments, and a 100\% concordance rate was achieved. Serum MAEA levels were measured using enzyme-linked immunosorbent assay (ELISA) kits (Westtang Biotech Inc., Shanghai, China) according to the manufacturer's protocol.

\section{Statistical analysis}

Student's $T$ tests were performed to compare demographic and characteristic variables between patients with PMOP and controls. Hardy-Weinberg equilibrium (HWE) tests were conducted for all 4 genotyped SNPs in the control group. Genetic association analyses were performed at both genotypic and allelic levels. $x^{2}$ tests were conducted, and odds ratios with $95 \%$ confidence intervals were calculated for each SNP. In addition to the single marker-based association analysis, we also constructed the linkage disequilibrium (LD) structure and examined the genetic association at the haplotype level. To investigate whether the genetic association signal obtained at the DNA level could be reflected at the protein level, we also analyzed the relationship between blood MAEA protein level and genotypes of significant SNPs. Analysis of variance (ANOVA) was used to test the statistical significance. Bonferroni correction was applied to address multiple comparisons. For single marker-based association analyses, the threshold of $P$ values was $0.05 / 4=0.0125$. Haploview was utilized for $L D$ structure construction [14]. Plink was used for HWE tests and genetic association analysis [15]. Statistical computing software $\mathrm{R}$ was utilized for other conventional statistical analyses [16].

\section{Bioinformatics analysis}

To examine the potential effects of significant SNPs on the gene expression of $M A E A$ in multiple types of human tissues, we conducted expression quantitative trait loci (eQTL) analysis based on data extracted from the Genotype-Tissue Expression (GTEx) database [17]. In addition, we also investigated other potential candidate genes by exploring the protein-protein interaction (PPI) network of MAEA using the STRING database [18].

\section{Results}

We examined the distributions of age, years to menopause, BMI and MAEA blood level between patients with PMOP and controls. The average blood level of
MAEA protein in patients with PMOP was significantly lower than that in controls $(T=-3.62, P=0.0003$, Table 1$)$. No significant differences were identified for age, BMI or years to menopause between patients with PMOP and controls.

All selected SNPs were in HWE in the control group (Supplemental Table S1). SNP rs6815464 was significantly associated with the risk of PMOP (Table 1). The $\mathrm{C}$ allele of rs6815464 was significantly associated with a decreased risk of PMOP in our study subjects (OR [95\% $C I]=0.75[0.63-0.89], P=0.0015$, Table 2). Dosage dependence patterns were identified through genotypic analyses. The OR for the CC genotype was 0.54 , and for the CT genotype it was 0.75 . In addition to significant signals identified in single marker-based association analyses, we also identified haplotypic association signals. One 2-SNP LD block was obtained (rs12641735rs6815464, Supplemental Figure S1), and further association analysis showed that this haplotype was significantly associated with PMOP $(P=0.0057$, Table 3$)$.

We further examined the differences in MAEA protein blood levels among genotypes of SNP rs6815464. Significant differences were identified in both the PMOP $(\mathrm{F}=$ 6.82, $P=0.0012)$ and control groups $(\mathrm{F}=11.5, P=0.00001)$ (Figure 1). As shown in Figure 1, the $C$ allele was associated with decreased MAEA protein levels in peripheral blood.

Genome-wide significant eQTL signals were identified for SNP rs6815464 on $M A E A$ in four types of human tissues, including whole blood $(P=1.70 \times 10-6)$, sun-exposed skin $(P=1.10 \times 10-5)$, tibial nerve $(P=2.80 \times 10-5)$ and thyroid $(P=5.00 \times 10-5)$. The directions of effect were the same in all four human tissues. The $\mathrm{C}$ allele of SNP rs6815464 was significantly associated with decreased MAEA gene expression (Table 4). A PPI network of $M A E A$ was constructed, and several closely connected genes, including GID4, LIX1, RMND5A, RMND5B, $A R M C 8$ and WDR26, were identified (Figure 2).

\section{Discussion}

Our results suggest that genetic polymorphisms of the $M A E A$ gene could contribute to the risk of PMOP in Chinese Han populations. In a cross-sectional study performed based on the Japanese population, Yulan et al. reported that the G allele carriers of SNP rs6815464 were significantly associated with low BMD of the hip

Table 1 Characteristic information of the study subjects

\begin{tabular}{lllll}
\hline Variables, mean \pm sd & Cases $(\mathbf{N}=\mathbf{4 8 4})$ & Controls $(\mathbf{N}=\mathbf{4 8 4})$ & T-Statistics & \multicolumn{1}{c}{$\boldsymbol{P}$-Values } \\
\hline Age, years & $62.8 \pm 6.9$ & $62.8 \pm 5.7$ & -0.10 & 0.9226 \\
Years to Menopause, years & $10.9 \pm 5.5$ & $11.1 \pm 5.4$ & -0.62 & 0.5361 \\
BMl, $\mathrm{kg} / \mathrm{m}^{2}$ & $23.2 \pm 1.6$ & $23.2 \pm 1.8$ & -0.28 & 0.7819 \\
MAEA level, $\mathrm{pg} / \mathrm{ml}$ & $915.9 \pm 263.1$ & $971.4 \pm 210.4$ & -3.62 & 0.0003 \\
\hline
\end{tabular}


Table 2 Results of the single marker based association analyses

\begin{tabular}{|c|c|c|c|c|c|c|c|c|c|c|c|c|c|c|}
\hline \multirow[t]{2}{*}{ CHR } & \multirow[t]{2}{*}{ SNP } & \multirow[t]{2}{*}{ POS } & \multicolumn{6}{|c|}{ Genotypic Analyses } & \multicolumn{6}{|c|}{ Allelic Analyses } \\
\hline & & & Genotypes & Cases & Controls & OR[95\%Cl] & $x^{2}$ & $P$-Values & Alleles & Cases & Controls & OR[95\%Cl] & $x^{2}$ & $P$-Values \\
\hline \multirow[t]{3}{*}{4} & rs12641735 & 1310646 & GG & 34 & 24 & $1.57[0.91-2.71]$ & & & & & & & & \\
\hline & & & CG & 184 & 166 & $1.49[0.94-1.60]$ & & & G & 252 & 214 & $1.24[1.00-1.53]$ & & \\
\hline & & & $\mathrm{CC}$ & 266 & 294 & ref & 4.05 & 0.1320 & C & 716 & 754 & ref & 4.08 & 0.0434 \\
\hline \multirow[t]{3}{*}{4} & rs6815464 & 1316113 & $\mathrm{CC}$ & 92 & 127 & $0.54[0.37-0.78]$ & & & & & & & & \\
\hline & & & CG & 254 & 254 & $0.75[0.55-1.02]$ & & & C & 438 & 508 & $0.75[0.63-0.89]$ & & \\
\hline & & & GG & 138 & 103 & ref & 10.68 & 0.0048 & G & 530 & 460 & ref & 10.13 & 0.0015 \\
\hline \multirow[t]{3}{*}{4} & rs72501966 & 1316879 & $\pi$ & 13 & 7 & $2.02[0.79-5.12]$ & & & & & & & & \\
\hline & & & $\mathrm{CT}$ & 155 & 134 & $1.26[0.95-1.66]$ & & & $\mathrm{T}$ & 181 & 148 & $1.27[1.00-1.62]$ & & \\
\hline & & & $\mathrm{CC}$ & 316 & 343 & ref & 4.43 & 0.1090 & C & 787 & 820 & ref & 3.99 & 0.0458 \\
\hline \multirow[t]{3}{*}{4} & rs10025665 & 1318479 & GG & 58 & 60 & $0.94[0.63-1.42]$ & & & & & & & & \\
\hline & & & AG & 213 & 216 & $0.96[0.74-1.26]$ & & & G & 329 & 336 & $0.97[0.80-1.17]$ & & \\
\hline & & & AA & 213 & 208 & ref & 0.11 & 0.9445 & A & 639 & 632 & ref & 0.11 & 0.7376 \\
\hline
\end{tabular}

CHR Chromosome, POS Position. Threshold of $P$-Values $=0.05 / 4=0.0125$. Significant results were highlighted in bold

but not of the lumbar spine or femoral neck [13]. On the other hand, in our study, the diagnosis of PMOP was based on the BMD of the lumbar spine or femoral neck. In this sense, our findings replicated and supplemented the results of a previous study based on Japanese populations. Despite the differences in sample size, sample ethnic ancestry, study design and the targeted phenotype between our study and the previous cross-sectional study, the direction of the effect direction of SNP rs6815464 is the same, and the effect size is quite similar. SNP rs6815464 located at the intronic region of gene MAEA with unclear functional consequence. Therefore in addition to simple genetic association analyses at the DNA level, in the present study, we moved further to investigate the association between genotypes of SNP rs6815464 and MAEA gene expression levels in several types of human tissues and blood levels of MAEA protein by integrating multiple lines of evidence.

Our findings in distributions of MAEA protein blood level indicated that the genotypes of SNP rs6815464 were significantly associated with the MAEA protein level in human blood in patients with PMOP and controls. With more copies of the $\mathrm{C}$ allele present, the individuals have lower MAEA protein levels on average in their blood samples. This finding could, at least partly, be confirmed by our eQTL data extracted from the GTEx database. In human tissue of whole blood, the C allele was significantly associated with a decreased level of $M A E A$ gene expression. It seems that the effect direction of SNP rs6815464 was the same at both the RNA and protein levels. Nevertheless, we need to be careful in interpreting these results, especially for those eQTL signals obtained from publicly available databases. The sources of the samples tested in the GTEx database were unknown, and therefore, we cannot confirm whether they are patients with OP. Since the patterns of gene expression could be very different in patients with PMOP and healthy people, the eQTL signals reported in the GTEx database might not be applicable to the present study.

Gene $M A E A$ encodes an integral membrane protein that mediates the attachment of erythroblasts to macrophages [19]. A recent study indicated that deletion of Maea in mouse macrophages caused severe reductions in bone marrow macrophages, erythroblasts, and in vivo island formation [19]. MAEA has an important role in hematopoiesis in BM, including granulocytemacrophage colony-forming units, which are the monocytes of osteoclasts [20]. In addition, a recent study also indicated that genetic polymorphisms of $M A E A$ contributed to the risk of periodontitis [21]. In the present study, the C allele of SNP rs6815464, which was associated with a decreased risk of PMOP, was associated with both lower RNA and protein levels in human blood.

Table 3 Results of haplotype based association analyses

\begin{tabular}{llllllll}
\hline LOCUS & HAPLOTYPE & F_A & F_U & $\mathbf{X}^{2}$ & DF & P-Values & SNPs \\
\hline H1 & OMNIBUS & - & - & 10.33 & 2 & 0.0057 & rs $12641735 \mid \mathrm{rs} 6815464$ \\
$\mathrm{H} 1$ & CC & 0.45 & 0.52 & 10.18 & 1 & 0.0014 & $\mathrm{rs} 12641735 \mid \mathrm{r} 6815464$ \\
$\mathrm{H} 1$ & GG & 0.26 & 0.22 & 4.19 & 1 & 0.0408 & $\mathrm{rs} 12641735 \mid \mathrm{rs} 6815464$ \\
$\mathrm{H} 1$ & $\mathrm{CG}$ & 0.30 & 0.26 & 2.62 & 1 & 0.1053 & $\mathrm{rs} 12641735 \mid \mathrm{r} 6815464$ \\
\hline
\end{tabular}

F_A: haplotype frequency in cases; F_U: haplotype frequency in controls; DF: degree of freedom 


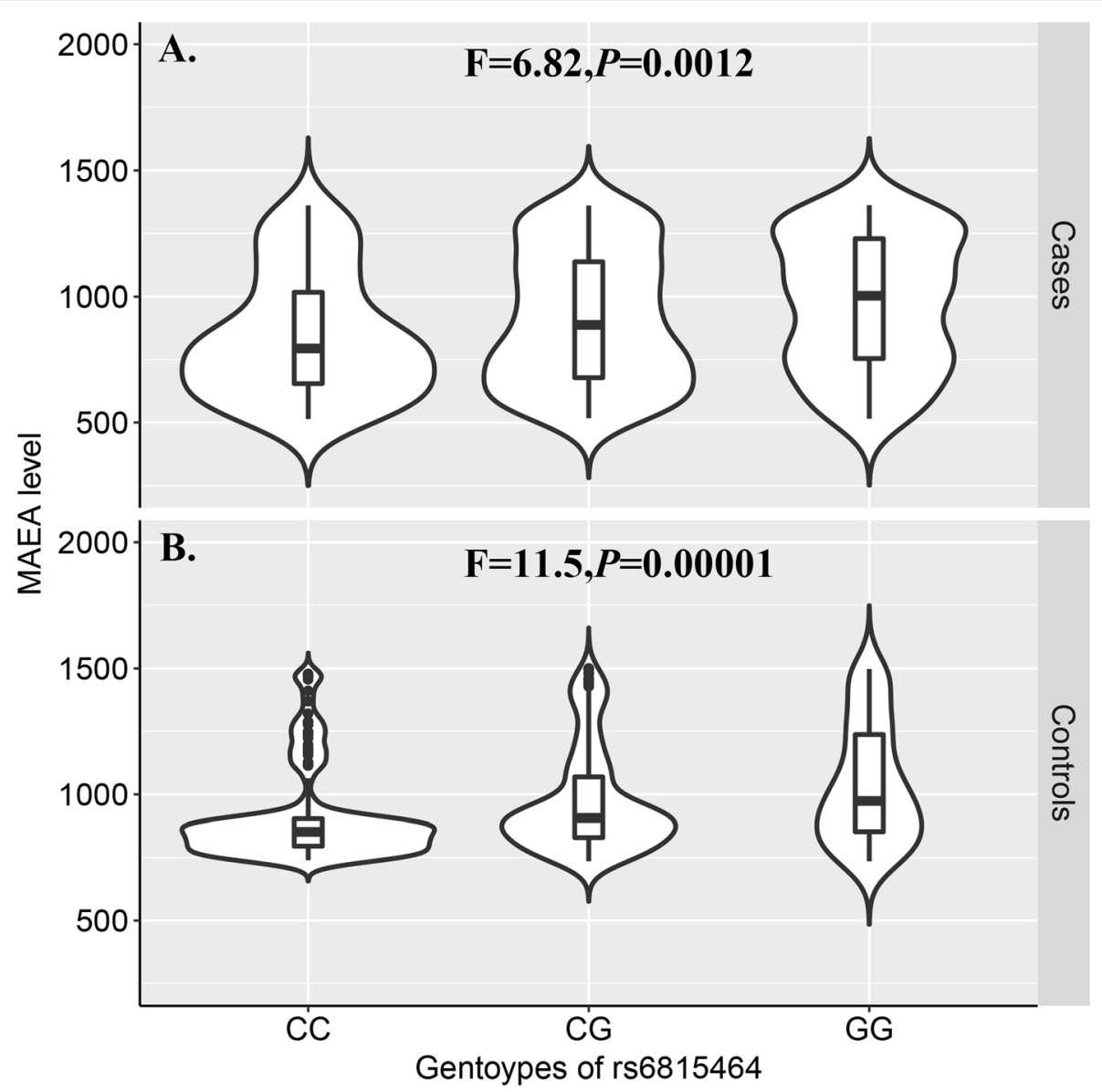

Fig. 1 Violin and boxplots for MAEA protein level in blood in the study subjects stratified by genotypes of SNP rs6815464. A. Cases. B. Controls. Fstatistics and $P$-Values were indicated

Based on these results, we hypothesized that MAEA might be involved in the genesis of osteoclasts. A reduction in MAEA expression might cause a decrease in BM macrophages and have a protective effect on PMOP through blocking the genesis of osteoclasts. Further functional analyses based on animal models are still needed to unravel the pathological mechanisms of MAEA protein and PMOP.

Despite the evidence that SNP rs6815464 is associated with gene expression level of $M A E A$ and blood level of
MAEA protein, it is too early to confirm that this SNP is the DNA variant with true biological effect. It is likely that SNP rs6815464 is just a surrogate of some other underlying DNA variants. These underlying DNA variants could be some other genetic polymorphisms that were not genotyped in the present study, or they could be some low-frequency or rare DNA variants that were not considered in the current study. Multiple lines of evidence have shown that these low-frequency and rare DNA variants might play an important role in the onset

Table 4 cis-eQTL signals of SNP rs6815464 that achieved genome-wide significance

\begin{tabular}{lllllll}
\hline SNP & Gene & Ref_Allele & Alt_Allele & P-Values & NES & Tissue \\
\hline rs6815464 & MAEA & C & $G$ & $1.70 \times 10^{-6}$ & -0.17 & Whole Blood \\
rs6815464 & MAEA & C & $G$ & $1.10 \times 10^{-5}$ & -0.31 & Skin - Sun Exposed (Lower leg) \\
rs6815464 & MAEA & C & $G$ & $2.80 \times 10^{-5}$ & -0.47 & Nerve - Tibial \\
rs6815464 & MAEA & C & $G$ & $5.00 \times 10^{-5}$ & -0.44 & Thyroid \\
rs6815464 & AC139887.4 & C & $G$ & $6.80 \times 10^{-5}$ & 1.10 & Brain - Cerebellum \\
rs6815464 & CTBP1 & C & $G$ & $7.80 \times 10^{-5}$ & 0.25 & Lung \\
rs6815464 & CTBP1-AS2 & C & $G$ & $1.10 \times 10^{-5}$ & -0.34 & Nerve - Tibial
\end{tabular}




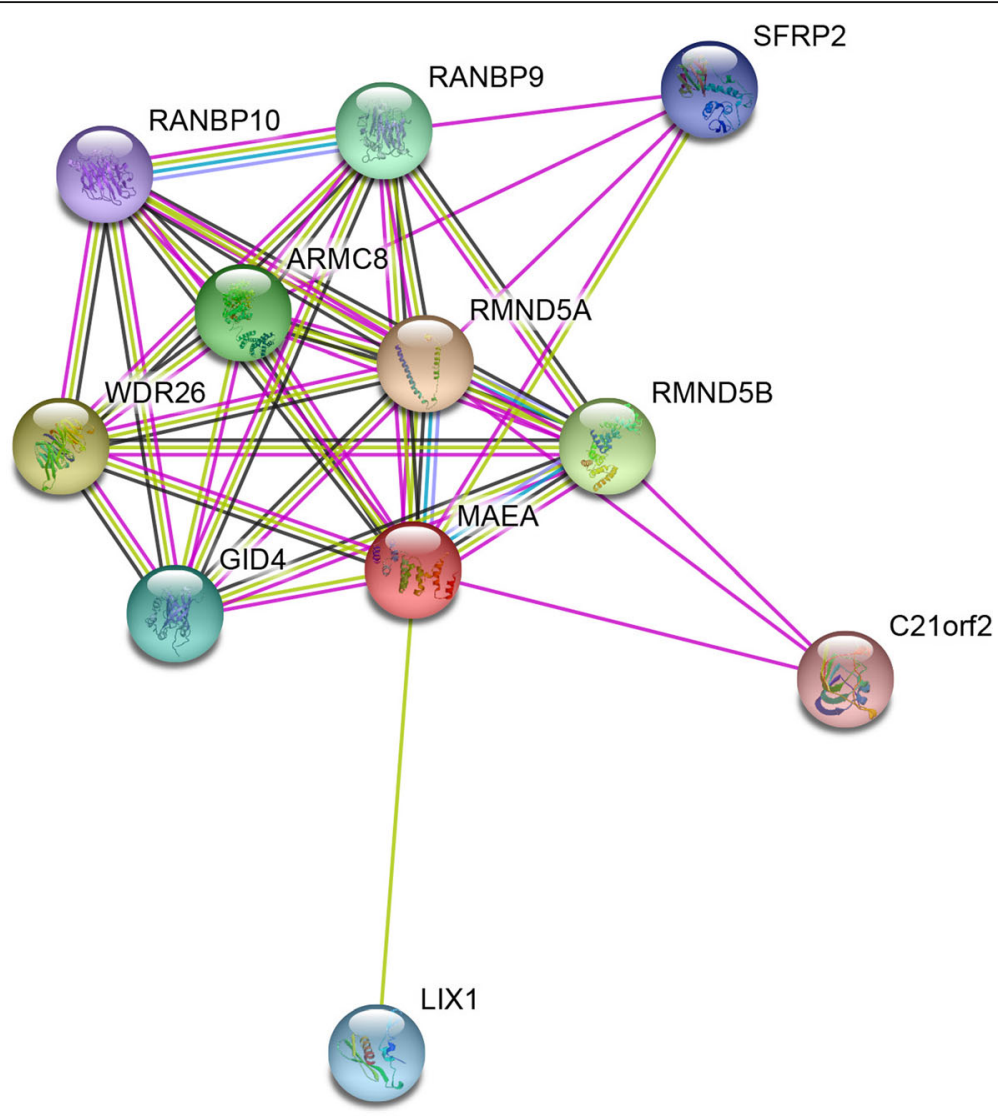

Fig. 2 Protein-protein interactions network for MAEA and its related proteins

and development of some complex disorders, including osteoporosis [22-25]. The genotyping technology and SNP selection strategy utilized in the present study cannot capture the information of these low-frequency DNA variants. Therefore, in the future, next-generation sequencing-based studies should be conducted to thoroughly investigate the genetic architecture of $M A E A$ and its effects on PMOP.

This study has several limitations. Only postmenopausal women were included as study subjects, although OP is a common disease in both senior men and women. This strategy accelerated the sample recruitment process because the incidence of $\mathrm{OP}$ in postmenopausal women is much higher than that in senior men. However, on the other hand, this strategy makes it more difficult to generalize our results to wider populations. Another limitation is that we did not perform any statistical procedures to adjust the potential population stratification. Population stratification is a potential confounder for genetic association mapping. As a candidate gene based study with only 4 SNPs genotyped, it is very difficult for us to adjust this confounder using some standard procedures such as principle component analysis or genomic control. Nevertheless, in the sample recruitment process, we restricted our study subjects by their immigration history to control their genetic backgrounds. We believe that this procedure would, at least partly, control the potential population stratification.

In summary, this case-control study based on Chinese postmenopausal women indicated an association signal between the GG genotype of rs6815464 in MAEA gene and the increased risk of PMOP. Further analyses showed that genotypes of SNP rs6815464 were also associated with the blood level of MAEA protein. These results suggested that rs6815464 in MAEA gene may be genetic factors affecting the risk of osteoporosis in Chinese postmenopausal women.

\section{Supplementary Information}

The online version contains supplementary material available at https://doi. org/10.1186/s13018-020-02140-4.

Additional file 1: Supplemental Tables S1. Genetic information of the 4 genotyped SNPS. Supplemental Figure S1. Linkage disequilibrium structure of the 4 genotyped SNPs. Values of D' were indicated in each cell. LD block was indicated by black bold frame.

\section{Abbreviations}

OP: Osteoporosis; BMD: Bone mineral density; GWAS: Genome-wide association study; MAEA: Macrophage erythroblast attacher; SNP: Single nucleotide polymorphism; PMOP: Postmenopausal osteoporosis; MAF: Minor 
allele frequency; ELISA: Enzyme-linked immunosorbent assay; HWE: HardyWeinberg equilibrium; LD: Linkage disequilibrium; ANOVA: Analysis of variance; eQTL: Expression quantitative trait loci; PPI: Protein-protein interaction; GTEx: Genotype-tissue expression; ORs: Odds ratios

\section{Acknowledgments}

We thank Dr. Fanglin Guan (Xi'an Jiaotong University Health Science Center) for his help and advice on the genetic statistics.

\section{Authors' contributions}

Authors Xijing He conceived and designed the study. Xuan Cai carried out candidate SNPs selection and statistical analyses. Xuan Cai, Jun Dong, Teng Lu and Liqiang Zhi conducted subject screening. Xuan Cai, Jun Dong and Teng Lu contributed to the collection and preparation of DNA samples. Xuan Cai wrote the paper. The authors read and approved the final manuscript.

\section{Funding}

This work was totally supported by the Fundamental Research Funds of Xi'an Jiaotong University (No. XJJ2018124). The funding sources had no role in the design of this study, the collection, analysis and interpretation of data, the writing of the report, or the decision to submit the paper for publication.

\section{Availability of data and materials}

Please contact the authors for reasonable requests.

\section{Ethics approval and consent to participate}

Written informed consent was obtained from all participants prior to their participation. The research protocol was approved by the Ethics Committee of the Second Affiliated Hospital of Xi'an Jiaotong University. The ethical approval was consistent with the standards of the Declaration of Helsinki.

\section{Consent for publication}

Not applicable.

\section{Competing interests}

None.

\section{Author details}

'Department of Orthopaedic Surgery, The Second Affiliated Hospital of Xi'an Jiaotong University, No. 157 Xiwu Road, Xi'an 710004, China. ${ }^{2}$ Department of Joint Surgery, Honghui Hospital of Xi'an Jiaotong University, No.555, Youyi East Road, Xi'an 710054, China.

Received: 18 June 2020 Accepted: 30 November 2020

Published online: 10 January 2021

\section{References}

1. Eastell R, O'Neill TW, Hofbauer LC, Langdahl B, Reid IR, Gold DT, et al. Postmenopausal osteoporosis. Nat Rev Dis Primers. 2016;2:16069. https://doi. org/10.1038/nrdp.2016.69.

2. Wade SW, Strader C, Fitzpatrick LA, Anthony MS, O'Malley CD. Estimating prevalence of osteoporosis: examples from industrialized countries. Arch Osteoporos. 2014;9:182. https://doi.org/10.1007/s11657-014-0182-3.

3. Boschitsch EP, Durchschlag E, Dimai HP. Age-related prevalence of osteoporosis and fragility fractures: real-world data from an Austrian menopause and osteoporosis clinic. Climacteric. 2017;20(2):157-63. https:// doi.org/10.1080/13697137.2017.1282452.

4. Alswat KA. Gender disparities in osteoporosis. J Clin Med Res. 2017:9(5):3827. https://doi.org/10.14740/jocmr2970w.

5. Noh JW, Park H, Kim M, Kwon YD. Gender differences and socioeconomic factors related to osteoporosis: a cross-sectional analysis of nationally representative data. J Womens Health (Larchmt). 2018;27(2):196-202. https:// doi.org/10.1089/jwh.2016.6244

6. Youness ER, Mohammed NA, Morsy FA. Cadmium impact and osteoporosis: mechanism of action. Toxicol Mech Methods. 2012;22(7):560-7. https://doi. org/10.3109/15376516.2012.702796

7. Eisman JA. Genetics of osteoporosis. Endocr Rev. 1999;20(6):788-804 https://doi.org/10.1210/edrv.20.6.0384.

8. Peacock M, Turner CH, Econs MJ, Foroud T. Genetics of osteoporosis. Endocr Rev. 2002;23(3):303-26. https://doi.org/10.1210/edrv.23.3.0464.
9. Ralston SH, Uitterlinden AG. Genetics of osteoporosis. Endocr Rev. 2010; 31(5):629-62. https://doi.org/10.1210/er.2009-0044.

10. Trajanoska K, Rivadeneira F. The genetic architecture of osteoporosis and fracture risk. Bone. 2019;126:2-10. https://doi.org/10.1016/j.bone.2019.04.005.

11. Francois S, Benmalek A, Guaydier-Souquieres G, Sabatier JP, Marcelli C. Heritability of bone mineral density. Rev Rhum Engl Ed. 1999;66(3):146-51.

12. Buniello A, MacArthur JAL, Cerezo M, Harris LW, Hayhurst J, Malangone C, et al. The NHGRI-EBI GWAS catalog of published genome-wide association studies, targeted arrays and summary statistics 2019. Nucleic Acids Res. 2019;47(D1):D1005-D12. https://doi.org/10.1093/nar/gky1120.

13. Che Y, Sugita N, Yoshihara A, Iwasaki M, Miyazaki H, Nakamura K, et al. A polymorphism rs6815464 in the macrophage erythroblast attacher gene is associated with low bone mineral density in postmenopausal Japanese women. Gene. 2019;700:1-6.

14. Barrett JC, Fry B, Maller J, Daly MJ. Haploview: analysis and visualization of LD and haplotype maps. Bioinformatics. 2005;21(2):263-5. https://doi.org/10. 1093/bioinformatics/bth457.

15. Chang CC, Chow CC, Tellier LC, Vattikuti S, Purcell SM, Lee JJ. Secondgeneration PLINK: rising to the challenge of larger and richer datasets. Gigascience. 2015;4:7. https://doi.org/10.1186/s13742-015-0047-8.

16. Team CR. R: a language and environment for statistical computing; 2019.

17. Consortium GT. The genotype-tissue expression (GTEx) project. Nat Genet. 2013;45(6):580-5. https://doi.org/10.1038/ng.2653.

18. Szklarczyk D, Gable AL, Lyon D, Junge A, Wyder S, Huerta-Cepas J, et al. STR NG v11: protein-protein association networks with increased coverage, supporting functional discovery in genome-wide experimental datasets. Nucleic Acids Res. 2019;47(D1):D607-D13. https://doi.org/10.1093/nar/ gky1131.

19. Wei $Q$, Boulais $P$, Zhang $D$, et al. Maea expressed by macrophages, but not erythroblasts, maintains postnatal murine bone marrow erythroblastic islands. Blood. 2019:133(11):1222-32.

20. Mao X, Shi X, Liu F, Li G, Hu L. Evaluation of erythroblast macrophage protein related to erythroblastic islands in patients with hematopoietic stem cell transplantation. Eur J Med Res. 2013;18:9. https://doi.org/10.1186/2047783X-18-9.

21. Che Y, Sugita N, Yoshihara A, Iwasaki M, Miyazaki H, Nakamura K, et al. MAEA rs6815464 polymorphism and periodontitis in postmenopausal Japanese females: a cross-sectional study. Arch Oral Biol. 2019;102:128-34. https://doi.org/10.1016/.archoralbio.2019.04.008.

22. Nielson CM, Zmuda JM, Carlos AS, Wagoner WJ, Larson EA, Orwoll ES, et al. Rare coding variants in ALPL are associated with low serum alkaline phosphatase and low bone mineral density. J Bone Miner Res. 2012;27(1): 93-103. https://doi.org/10.1002/jbmr.527.

23. Zwick MEME. Evaluating rare variants in complex disorders using nextgeneration sequencing. Curr Psychiatry Rep. 2013;15(4):1-7.

24. Kemp JP, Morris JA, Medina-Gomez C, Forgetta V, Warrington NM, Youlten SE, et al. Identification of 153 new loci associated with heel bone mineral density and functional involvement of GPC6 in osteoporosis. Nat Genet. 2017:49(10):1468-75. https://doi.org/10.1038/ng.3949.

25. Zhang T, Hou L, Chen DT, McMahon FJ, Wang JC, Rice JP. Exome sequencing of a large family identifies potential candidate genes contributing risk to bipolar disorder. Gene. 2018;645:119-23. https://doi.org/ 10.1016/j.gene.2017.12.025

\section{Publisher's Note}

Springer Nature remains neutral with regard to jurisdictional claims in published maps and institutional affiliations.

Ready to submit your research? Choose BMC and benefit from:

- fast, convenient online submission

- thorough peer review by experienced researchers in your field

- rapid publication on acceptance

- support for research data, including large and complex data types

- gold Open Access which fosters wider collaboration and increased citations

- maximum visibility for your research: over $100 \mathrm{M}$ website views per year

At $\mathrm{BMC}$, research is always in progress.

Learn more biomedcentral.com/submission 